\title{
PC12细胞低压性缺氧损伤模型的建立
}

张冬梅 ${ }^{1}$, 曹琪璐 ${ }^{1}$, 景临林 $^{1}$, , 赵秀华 $^{2}$, 马慧萍 ${ }^{1}$

1. 中国人民解放军联勤保障部队第九四○医院药剂科全军高原医学实验室, 甘肃 兰州 730050

2. 中国人民解放军西部战区疾病预防控制中心传染病防控二科, 四川成都 610000

[摘要] 目的: 构建 PC12 细胞低压性缺氧细胞模型。方法: 将PC12 细胞依据缺 氧条件随机分为正常对照组、常压缺氧组、低压缺氧组; 正常对照组以正常条件 培养, 常压缺氧组和低压缺氧组分别以常压缺氧和低压缺氧条件培养。通过考察 氧气浓度、大气压力和低压缺氧时间, 应用 CCK-8 法测定细胞活性, 篮选低压缺氧 的条件; 显微镜下观察各组细胞形态、结构、数目, 微板法检测细胞乳酸脱氢酶 ( $\mathrm{LDH})$ 、超氧化物歧化酶 $(\mathrm{SOD})$ 活性和丙二醛 $(\mathrm{MDA})$ 含量, 流式细胞仪测定细胞调 亡比例和细胞周期。结果: 在 $1 \%$ 氧浓度和 $41 \mathrm{kPa}$ 低压缺氧环境下培养 $24 \mathrm{~h}$ 可建立 低压性缺氧细胞模型。与正常对照组和常压缺氧组比较, 低压缺氧组 LDH 活性及 细胞MDA含量增加、SOD活性下降, 细胞调亡比例上升 (均 $P<0.05$ ), 细胞周期阻 滞发生在 G0/G1 期。结论: 建立了稳定可靠的低压性缺氧损伤 PC12 细胞模型, 可 用于高原低压性缺氧神经损伤的体外实验研究。

[关键词 ] PC12 细胞; 低压性缺氧; 乳酸脱氢酶; 超氧化物歧化酶; 丙二醛; 细胞凋 亡; 细胞周期

[中图分类号 ］R966 [ 文献标志码 ］A

\section{Establishment of a hypobaric hypoxia-induced cell injury model in PC12 cells}

ZHANG Dongmei ${ }^{1}$, CAO Qilu ${ }^{1}$, JING Linlin ${ }^{1}$, ZHAO Xiuhua ${ }^{2}$, MA Huiping ${ }^{1}$ (1. Key Laboratory of the Plateau Medicine, the 940th Hospital of Joint Logistics Support Force of Chinese People's Liberation Army, Lanzhou 730050, China; 2. Second Department of Infectious Disease Control and Prevention, Center for Disease Control and Prevention, Western War Zone, Chengdu 610000, China)

Corresponding authors: MA Huiping, E-mail: 1026573411@qq.com, https://orcid.org/ 0000-0003-4560-2155; ZHAOXiuhua,E-mail: zhaoxiuhua2021xz@163.com, https://

\footnotetext{
收稿日期:2021-02-20 接受日期:2021-08-10

基金项目:国家自然科学基金(81571847,81872796); 军队后勤科研计划(CWH17J010,CLB18J028)

第一作者:张冬梅,硕士,主管药师,主要从事高原缺氧损伤分子机制研究;E-mail:835935150@qq.com; https://orcid.org/ 0000-0002-5708-8387

通信作者: 马慧萍,主任药师,教授,硕士生导师, 主要从事高原缺氧损伤机制及新药研发工作;E-mail: 1026573411@qq. com; https ://orcid.org/0000-0003-4560-2155. 赵秀华,副主任技师,主要从事高原疾病防控和实验工作;E-mail： zhaoxiuhua2021xz@163.com; https://orcid.org/0000-0002-3522-0930
} 
orcid.org/0000-0002-3522-0930

[ Abstract ] Objective: To construct a hypobaric hypoxia-induced cell injury model.

Methods: Rat pheochromocytoma PC12 cells were randomly divided into control group, normobaric hypoxia group and hypobaric hypoxia group. The cells in control group were cultured at normal condition, while cells in other two groups were cultured in normobaric hypoxia and hypobaric hypoxia conditions, respectively. CCK-8 method was used to detect cell viability to determine the optimal modeling conditions like the oxygen concentration, atmospheric pressure and low-pressure hypoxia time. The contents of lactate dehydrogenase (LDH), superoxide dismutase (SOD) and malondialdehyde (MDA) were detected by microplate method. The apoptosis ratio and cell cycle were analyzed by flow cytometry. Results: The hypobaric hypoxia-induced cell injury model can be established by culturing for $24 \mathrm{~h}$ at $1 \%$ oxygen concentration and $41 \mathrm{kPa}$ atmospheric pressure. Compared with the control group and normobaric hypoxia group, the activity of LDH and the content of MDA in hypobaric hypoxia group were significantly increased, the activity of SOD was decreased, the percentage of apoptosis was increased (all $P<0.05$ ), and the cell cycle was arrested in G0/G1 phase. Conclusion: A stable and reliable cell injury model induced by hypobaric hypoxia has been established with PC12 cells, which provides a suitable cell model for the in vitro experimental study on nerve injury induced by hypoxia at high altitude.

[ Key words ] PC12 cells; Hypobaric hypoxia; Lactate dehydrogenase; Superoxide dismutase; Malondialdehyde; Apoptosis; Cell cycle

[J Zhejiang Univ (Med Sci), 2021, 50(5): 614-620.]

[缩略语] 乳酸脱氢酶(lactate dehydrogenase, LDH); 超氧化物歧化酶( superoxide dismutase, SOD); 丙二醛 (malondialdehyde, MDA); 细胞计数试剂盒 (cell counting kit, CCK); 异硫氧酸荧光素(fluorescein isothiocyanate,FITC); 碘化丙啶(propidium iodide, PI); 磷酸盐缓冲液 (phosphate buffer solution,PBS)

机体脑组织对缺氧较为敏感, 当海拔上升到 一定高度后, 首先会出现缺氧性损害。而神经是 高分化细胞, 其结构及功能损伤后不可再生, 这也 是高原低氧环境致缺氧性脑损害的重要发病机制 之一 ${ }^{[1-2]}$ 。因此, 研究高原环境对神经细胞损伤的 影响是预防和治疗高原脑病的关键。目前高原缺 氧研究采用的模型主要是通过调控细胞培养环境 中氧分压或细胞利用氧的能力而建立细胞缺氧性 损伤模型,但这些建模方法不能模拟由于低大气 压力导致氧分压降低而造成的高原缺氧。本研究 采用降低氧气体积比同时模拟高海拔大气压力的 方法建立PC12 细胞高原低压缺氧损伤模型, 通过 笁选氧气浓度、大气压力和低压缺氧时间确定最
佳的造模条件,并通过观察该条件下细胞的形态, 测定细胞培养基中 $\mathrm{LDH} 、 \mathrm{SOD}$ 活性及 MDA 含量, 测定细胞周期和细胞凋亡情况, 来验证低压缺氧 损伤模型, 以期为高原神经损伤相关疾病的研究 建立一种稳定可靠的细胞模型。

\section{1 材料与方法}

\section{1 细胞、试剂及仪器}

PC12 细胞购自中国科学院上海细胞库。 DMEM 高糖培养基、青一链霉素为美国 HyClone公 司产品; 胎牛血清为美国 Gibco公司产品;LDH 试剂 盒(批号 20180728)、SOD试剂盒(批号 20190412)、 MDA 试剂盒(批号 20180728) 均为南京建成生物工 
程研究所产品;CCK-8 为日本同仁化学研究所产 品;FITC-Annexin V/PI 细胞调亡检测试剂盒为美国 $\mathrm{BD}$ 公司产品; 细胞周期检测试剂盒为碧云天生物 技术有限公司产品。

便携式细胞低氧小室 (mic-101) 为美国 Billups-Rothenberg 公司产品; 氧浓度测定仪(EST10-02)为美国 Estesnros 公司产品; 压力控制型细 胞缺氧培养箱为实验室自制 (实用新型专利号 CN208995505U); 倒置相差显微镜为日本 Olympus 公司产品; 三气培养箱为德国 Memmert 公司产品; 超净工作台 (SW-CJ-2FD) 为杭州净化工作设备厂 产品; Scotsman 制冰机 (AF 80)、全自动荧光酶标仪 (SpectraMax i3) 为美国 Molecular Devices 公司产 品; 流式细胞仪为美国 BD公司产品。

\section{$1.2 \mathrm{PC} 12$ 细胞培养}

PC12 细胞置于 DMEM 高糖培养液中, 培养 液含 $10 \%$ 胎牛血清、青霉素 $100 \mathrm{U} / \mathrm{mL}$ 、链霉素 $100 \mathrm{mg} / \mathrm{L}$, 于 $37^{\circ} \mathrm{C} 、 5 \%$ 二氧化碳培养箱内培养, 培 养液每2 3 d 更换一次。PC12 细胞进人对数生长 期后传代, 以 $1 \times 10^{5} / \mathrm{mL}$ 密度接种于底面积为 $10 \mathrm{~cm}^{2}$ 的塑料培养血中, $24 \mathrm{~h}$ 后更换培养液, 并根 据实验要求给予不同的培养条件。

\section{3 实验参数筛选}

1.3.1 氧气浓度耖选调整 PC12 细胞密度至 $5 \times 10^{4} / \mathrm{mL}$, 按 $100 \mu \mathrm{L} /$ 孔平行接种于 7 块 96 孔板 中, 每块 96 孔板设 6 个复孔, 于 $5 \%$ 二氧化碳、3 $3{ }^{\circ} \mathrm{C}$ 的三气培养箱中培养过夜。将 7 块 96 孔板随机分 为正常对照组以及不同氧气浓度的常压缺氧组, 正常对照组在 $5 \%$ 二氧化碳、3 ${ }^{\circ} \mathrm{C}$ 三气培养箱内正 常培养; 常压缺氧组放至缺氧小室, 分别用 $5 \%$ 二氧 化碳、一定浓度氮气和氧气的混合气体 (二氧化碳 比例保持在 $5 \%$, 氮气比例随氧气浓度变化而变化, 下同)置换其中空气, 应用氧浓度测定仪测定氧浓 度使之终浓度分别为 $0 \% 、 1 \% 、 2 \% 、 3 \% 、 4 \% 、 5 \%$, 放 人培养箱中培养 $24 \mathrm{~h}$ 。应用CCK-8法测定各组细 胞活性, 选择细胞活性明显降低的氧浓度进行后 续实验。

1.3.2 大气压力笁选 按照 1.3.1节中的方法, 将PC12 细胞平行接种于 7 块 96 孔板中, 正常培养 $12 \mathrm{~h}$ 后将细胞随机分为正常对照组、常压缺氧组和 不同压力的低压缺氧组。正常对照组正常培养; 常 压缺氧组移入缺氧小室, 用混合气体置换其中空气 使氧气终浓度为 $1 \%$, 将缺氧小室放于三气培养箱
继续培养 $24 \mathrm{~h}$; 低压缺氧组将培养血置于低压缺氧 装置中, 用混合气体置换其中空气使氧气终浓度为 $1 \%$, 调整使用不同压力 $(41 、 47 、 54 、 62 、 71 \mathrm{kPa})$, $24 \mathrm{~h}$ 后应用CCK-8 法检测各组细胞活性, 选择细胞 活性明显降低的压力进行后续实验。

1.3 .3 低压缺氧时间的笁选按照1.3.1节中 的方法, 将PC12 细胞分 5 个时间点平行接种于 15 块 96 孔板中 (每次接种三块板), 正常培养 $12 \mathrm{~h}$ 后 将每个时间点的细胞均随机分为正常对照组、常 压缺氧组和低压缺氧组。正常对照组正常培养; 常压缺氧组移人缺氧小室中用混合气体置换其中 空气使氧气终浓度为 $1 \%$; 低压缺氧组置于低压缺 氧装置中, 以 $1 \%$ 氧浓度和 $41 \mathrm{kPa}$ 压力培养, 分别培 养 $6 、 12 、 24 、 48 、 72 \mathrm{~h}$ 后, 应用 CCK-8 法检测细胞 活性。

\subsection{CCK-8 法检测细胞活性}

细胞培养后, 在 96 孔板中每孔加人 $10 \mu \mathrm{L}$ CCK-8 试剂, 摇匀后置于 $5 \%$ 二氧化碳、 $37{ }^{\circ} \mathrm{C}$ 三气 培养箱中继续培养 $1 \mathrm{~h}$, 在酶标仪上测定 $450 \mathrm{~nm}$ 处 的吸光度值并计算细胞活性, 细胞活性 $(\%)=($ 实 验组吸光度值一空白吸光度值)/(正常对照组吸 光度值－空白吸光度值 $) \times 100 \%$ 。

\section{5 光镜下观察 $\mathrm{PC} 12$ 细胞形态}

将PC12 细胞以 $1 \times 10^{5} / \mathrm{mL}$ 密度接种于 $60 \mathrm{~mm}$ 培养血, 培养 $24 \mathrm{~h}$ 待细胞完全贴壁后, 分为正常对 照组、常压缺氧组和低压缺氧组。正常对照组正 常培养; 常压缺氧组在 $1 \%$ 氧浓度下培养; 低压缺 氧组在 $1 \%$ 氧浓度和 $41 \mathrm{kPa}$ 压力下培养。细胞培养 $24 \mathrm{~h}$ 后显微镜下拍照, 观察低压缺氧对 PC12 细胞 形态的影响。

\section{6 微板法测定细胞LDH、SOD 活性及 MDA 含量}

调整PC12 细胞密度至 $5 \times 10^{4} / \mathrm{mL}$, 按 $100 \mu \mathrm{L} /$ 孔接种于 96 孔板, 于 $5 \%$ 二氧化碳、37 ${ }^{\circ} \mathrm{C}$ 孵箱中培 养过夜。按 1.5 节中的培养方法将 96 孔板随机分 为正常对照组、常压缺氧组和低压缺氧组。细胞培 养 $24 \mathrm{~h}$ 后, 收集细胞上清液于新的 96 孔板中, 根据 说明书操作步骤分别测定 LDH、SOD 的活性及 MDA 含量。每组设 6 个复孔, 实验重复三次。

1.7 流式细胞术检测细胞调亡和细胞周期

将对数生长期的 PC12 细胞以 $1 \times 10^{5} / \mathrm{mL}$ 的密 度接种于 3 块六孔板中, 贴壁后按照 1.5 节中的培 养方法随机分为正常对照组、常压缺氧组和低压 缺氧组。细胞培养 $24 \mathrm{~h}$ 后, 弃去培养液, 用 $4{ }^{\circ} \mathrm{C}$ 预 
冷的 PBS 洗两次去掉残余培养基, 用不含乙二胺 四乙酸的胰蛋白酶消化, 室温下 $1500 \times g$ 离心 10 min,弃去培养基,收集细胞。

检测细胞凋亡: 用 $4{ }^{\circ} \mathrm{C}$ 预冷的 $1 \times \mathrm{PBS}$ 洗涤细 胞两次, $150 \times g$ 离心 $5 \mathrm{~min}$, 去上清液, 收集细胞; 加 人 $1 \times$ 结合缓冲液 $300 \mu \mathrm{L}$ 悬浮细胞, 调整细胞浓度 为 $1 \times 10^{6} / \mathrm{mL}$; 每 $100 \mu \mathrm{L}$ 细胞悬液加人 $5 \mu \mathrm{L}$ FITCAnnexin V 混匀, 室温避光捊育 $15 \mathrm{~min}$; 上机检测前 $5 \min$ 加人 $5 \mu \mathrm{L}$ PI染液; 加人 $400 \mu \mathrm{L} 1 \times$ 结合缓冲 液轻轻混匀; 将细胞转人流式管中, 应用流式细胞 仪检测。

检测细胞周期: 收集各组细胞于离心管中, $1500 \times g$ 离心 $5 \mathrm{~min}$, 收集细胞, PBS 洗两遍, 用 $70 \%$ 乙醇固定过夜, $1500 \times g$ 离心 $5 \mathrm{~min}$ 收集细胞, PBS洗 两遍, 使用 $100 \mu \mathrm{L}$ 核糖核酸酶 $\mathrm{A}$ 重悬细胞, 置于 $37^{\circ} \mathrm{C}$ 卿育 $30 \mathrm{~min}$, 加人 $400 \mu \mathrm{L}$ PI 染液, $4^{\circ} \mathrm{C}$ 条件下 避光捊育 $30 \mathrm{~min}$ 。应用细胞篎过滤细胞后, 将细胞 转人流式管中,应用流式细胞仪检测。

\section{8 统计学方法}

采用SPSS 22.0 软件进行统计分析。正态分 布的计量数据以均数 \pm 标准差 $(\bar{x} \pm s)$ 表示, 组间 两两比较采用单因素方差分析, $P<0.05$ 为差异有 统计学意义。

\section{2 结 果}

\section{1 氧气浓度对细胞活性的影响}

当氧浓度为 $0 \% 、 1 \% 、 2 \% 、 3 \%$ 时, 细胞活性较 正常对照组均下降 (均 $P<0.01$ ), 其中 $1 \%$ 氧浓度较 $2 \% 、 3 \%$ 氧浓度细胞活性下降( 均 $P<0.01$ ) , $0 \%$ 氧浓 度较 $1 \%$ 氧浓度细胞活性下降, 而当氧浓度为 $4 \%$ 、 $5 \%$ 时, 细胞活性与正常对照组接近 (均 $P>0.05$ ), 见图 1 。根据实验结果, 选择 $1 \%$ 氧浓度作为后续 缺氧模型的条件。

\section{2 大气压力对细胞活性的影响}

在 $1 \%$ 氧浓度下, 当压力为 $71 、 62$ 和 $54 \mathrm{kPa}$ 时 不足以造成细胞低压性缺氧损伤; 而当压力为 $41 、 47 \mathrm{kPa}$ 时细胞活性较常压缺氧组下降 (均 $P<0.05$ ), 其中 $41 \mathrm{kPa}$ 导致细胞活性较 $71 、 62$ 和 $54 \mathrm{kPa}$ 均下降 $($ 均 $P<0.05)$, 见图 2 。根据实验结 果, 选择 $41 \mathrm{kPa}$ 压力条件进行后续研究。

\section{3 低压缺氧时间对细胞活性的影响}

常压缺氧组和低压缺氧组在 5 个时间点的细 胞活性均比正常对照组降低 (均 $P<0.01)$ 。培养

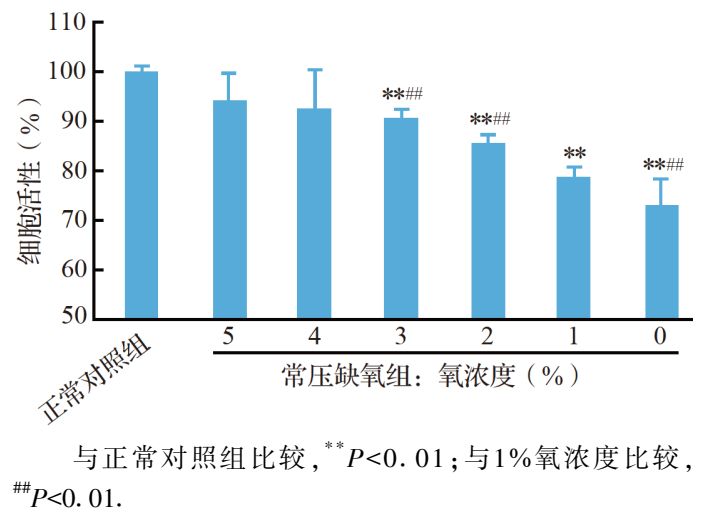

图 1 不同氧浓度细胞活性比较

Figure 1 Effects of different hypoxia concentrations on the activity of PC12 cells

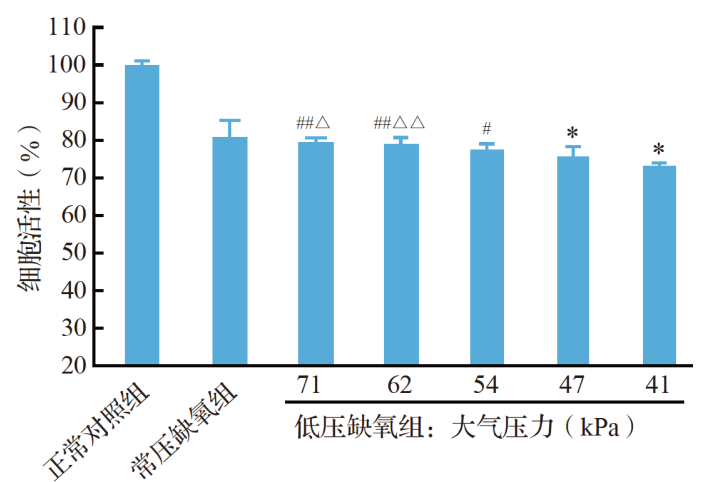

与常压缺氧组比较, ${ }^{*} P<0.05$; 与 $41 \mathrm{kPa}$ 压力比较, ${ }^{\#} P<0.05,{ }^{\#} P<0.01$; 与47 kPa压力比较, ${ }^{\triangle} P<0.05,{ }^{\triangle}{ }^{\triangle} P<0.01$.

图 $21 \%$ 氧浓度下不同大气压力细胞活性比较

Figure 2 Effects of atmospheric pressure on PC12 cell activity under $1 \%$ oxygen concentration

$6 、 12 \mathrm{~h}$, 低压缺氧组与常压缺氧组的细胞活性差异 无统计学意义 (均 $P>0.05$ ); 培养 $24 、 48 、 72 \mathrm{~h}$, 低压 缺氧组的细胞活性较常压缺氧组下降 (均 $P<$ 0.01 ), 见图3。结果提示, $1 \%$ 氧浓度和 $41 \mathrm{kPa}$ 压力 条件下培养 $24 \mathrm{~h}$ 即可造成细胞损伤。

\section{4 低压缺氧对细胞形态的影响}

正常对照组细胞贴壁生长状态良好, 边缘清 晰可见,多数伸出轴突, 细胞呈梭形状态; 常压缺 氧组细胞形态固缩, 伸出轴突的细胞数量减少, 多 数细胞成圆形, 细胞膜较粗粘, 细胞贴壁性较差; 低压缺氧组细胞肿胀, 死细胞增多, 细胞生长停 滞。见图4。结果提示, 低压缺氧环境比单纯低氧 环境可以造成更严重的细胞损伤。

2.5 低压缺氧对细胞上清液中 LDH 活性及细胞 中MDA 含量和SOD活性的影响

与正常对照组比较, 常压缺氧组和低压缺氧 


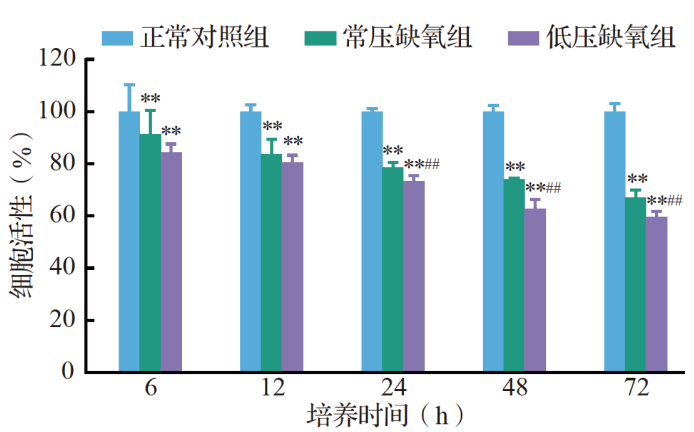
${ }^{\#} P<0.01$.

与正常对照组比较, ${ }^{* *} P<0.01$; 与常压缺氧组比较,

图 3 低压缺氧条件下不同培养时间点细胞活性比较

Figure 3 Effects of different culture time on PC12 cell activity under hypobaric hypoxia condition

组 $L D H$ 活性均升高 $($ 均 $P<0.05$ ), 且低压缺氧组 LDH 活性较常压缺氧组升高 $(P<0.05)$ 。与正常对 照组比较, 常压缺氧组和低压缺氧组 SOD 活性降 低, MDA 含量增加 (均 $P<0.05)$; 且与常压缺氧组 比较, 低压缺氧组 SOD 活性降低, MDA 含量增加 $($ 均 $P<0.05)$ 。见表 1 。结果提示, 与常压缺氧组比 较, 低压缺氧组细胞膜损伤程度加重、脂质过氧化 反应增加、抗氧化能力降低。

2.6 低压缺氧对细胞凋亡及细胞周期的影响 与正常对照组细胞凋亡率 $(6.40 \pm 0.53) \%$ 比
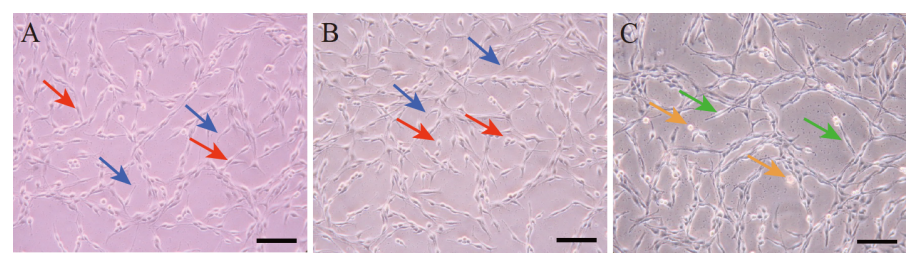

A:正常对照组细胞贴壁生长状态良好, 边缘清晰可见, 多数伸出轴突 (蓝 色箭头所示), 呈梭形状态 (红色箭头所示) ; B : 常压缺氧组细胞形态固缩, 伸出 轴突的细胞数量减少 (蓝色箭头所示), 多数细胞成圆形 (红色箭头所示), 细胞 膜较粗粘, 细胞贴壁性较差; C: 低压缺氧组细胞肿胀 (绿色箭头所示), 死细胞 增多 (橙色箭头所示), 细胞生长停滞. 标尺 $=100 \mu \mathrm{m}$.

图 4 各组细胞培养 $24 \mathrm{~h}$ 后光镜下形态

Figure 4 Morphology of PC12 cells cultured under different conditions for $24 \mathrm{~h}$
较, 常压缺氧组细胞凋亡率 $(14.50 \pm 0.95) \%$ 升高 $(P<0.05)$; 而低压缺氧组细胞调亡率 $(18.98 \pm$ $1.12) \%$ 较常压缺氧组进一步升高 $(P<0.01)$, 见图 5。与正常对照组比较, 常压缺氧组 $\mathrm{S}$ 期细胞 比例增加, 细胞被阻滞在 $\mathrm{S} / \mathrm{G} 2$ 期; 低压缺氧组 $\mathrm{G} 1$ 期的细胞比例增加,进人 $\mathrm{G} 2 / \mathrm{M}$ 期的细胞比例减 少。见图6。结果提示, 细胞在低压缺氧条件下培 养 $24 \mathrm{~h}$ 后, 增殖周期被阻滞在 G0/G1 期,进人DNA 复制期的细胞减少, 细胞增殖减慢甚至停滞, 细胞 调亡情况也较常压缺氧组更严重。

\section{3 讨 论}

相对于实验动物而言, 细胞在培养过程中具 有易获得、研究条件人为可控、研究样本均一、有 利于观察单一因素等优势 ${ }^{[3]}$ 。原代细胞更接近在 体状态，但原代培养成功率低、成本高、操作困难， 且在细胞培养过程中容易自然老化从而导致无法 持续传代。PC12 细胞源于大鼠肾上腺嗜铬细胞 瘤,在形态、生理及生化功能等方面接近于神经细 胞, 且具有可传代特点, 已经成为离体研究神经细 胞功能、死亡方式及毒性损害较理想的工具细 胞。研究高原低氧对 PC12 细胞的损害作用对 高原低氧致神经细胞损害的机制探索有重要 意义。

目前用于研究高原缺氧的细胞模型 主要是通过调控细胞培养环境中的氧分 压或细胞利用氧的能力而建立细胞缺氧 性损伤模型。离体细胞缺氧模型的建立 大体上分为物理性缺氧和化学性缺氧两 类 ${ }^{[4-9]}$ 。化学性缺氧模型是在细胞培养 液中加人特定的化学物质, 通过损伤细 胞利用氧的能力或耗尽培养液中的氧而 造成细胞缺氧。常用于建立化学性缺氧 模型的化学药物有氰化物、连二亚硫酸 钠、氯化钴等。化学性缺氧可能存在对

表 1 各组乳酸脱氢酶活性、丙二醛含量和超氧化物歧化酶活性比较

Table 1 Lactate dehydrogenase activity, malondialdehyde content and superoxide dismutase activity in each group

\begin{tabular}{ccccc} 
& & & $(\bar{x} \pm s)$ \\
\hline 组 别 & $n$ & 乳酸脱氢酶 $(\mathrm{U} / \mathrm{mL})$ & 丙二醛 $(\mathrm{nmol} / \mathrm{mg})$ & 超氧化物歧化酶 $(\mathrm{U} / \mathrm{mg})$ \\
\hline 正常对照组 & 6 & $22.55 \pm 2.11$ & $0.0345 \pm 0.0011$ & $0.123 \pm 0.101$ \\
常压缺氧组 & 6 & $38.15 \pm 1.99^{*}$ & $0.0683 \pm 0.0054^{*}$ & $0.116 \pm 0.083^{*}$ \\
低压缺氧组 & 6 & $43.15 \pm 3.25^{* * *}$ & $0.0789 \pm 0.0019^{* * * \#}$ & $0.112 \pm 0.052^{* * * \#}$ \\
\hline
\end{tabular}

与正常对照组比较, ${ }^{*} P<0.05,{ }^{* *} P<0.01 ;$ 与常压缺氧组比较, ${ }^{\#} P<0.05$. 

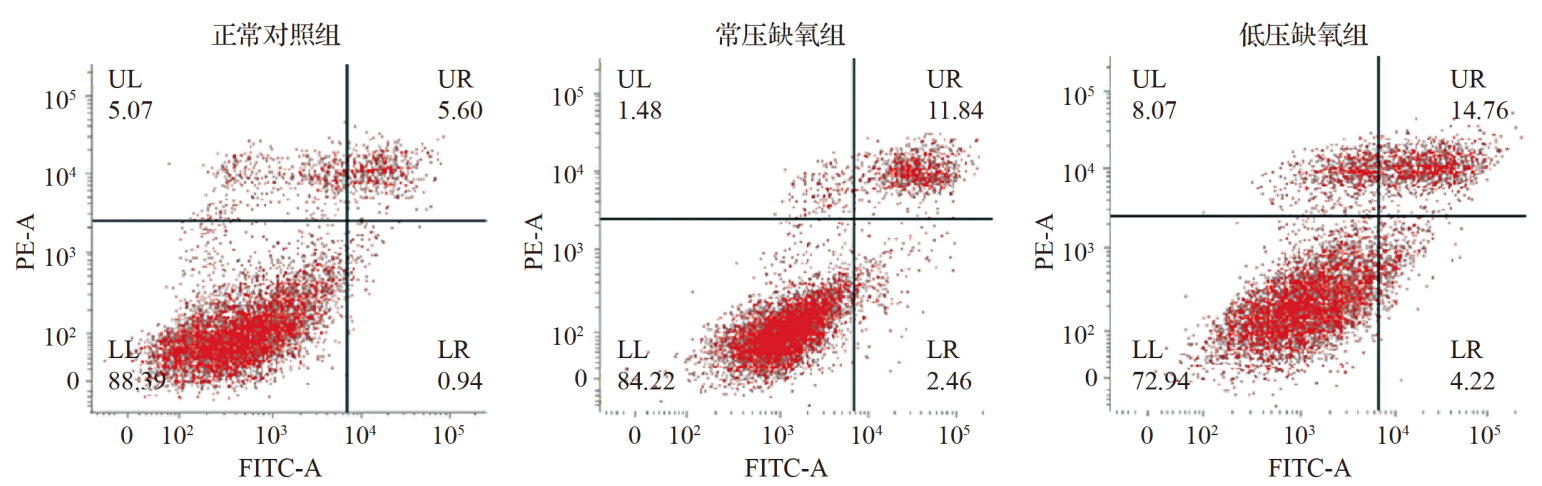

图 5 流式细胞术检测各组细胞凋亡结果

Figure 5 Results of apoptosis detected by flow cytometry
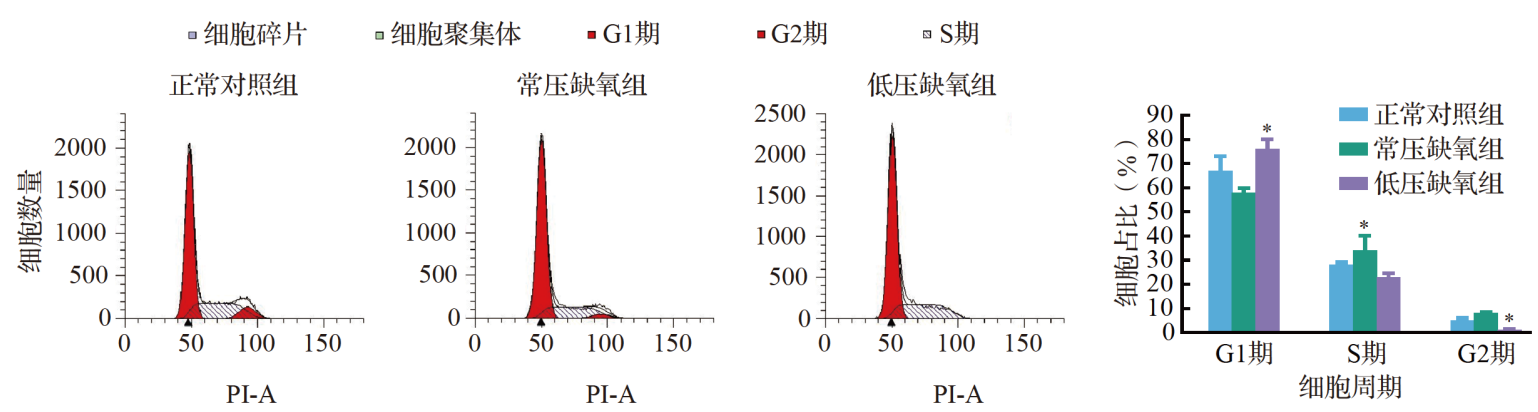

与正常对照组比较, ${ }^{*} P<0.05$.

图 6 各组细胞周期检测结果及比较

Figure 6 Cell cycles of PC12 cells in each group

细胞造成损伤、实验操作不易控制以及与实际环境 差异较大等缺点。物理性缺氧模型通常利用混合 气体培养法即利用降低培养环境中的氧气比例从 而模拟细胞缺氧损伤过程, 是目前较为常用的体外 缺氧实验模型, 通常有液面封闭模型、自制简易密 封舱模型及三气培养箱模型, 建立的模型比较稳 定。但以上细胞建模方法不能模拟由于高原环境 低大气压力导致氧分压降低造成的高原缺氧, 而压 力对细胞的贴壁以及增殖均有一定影响 ${ }^{[10-12]}$, 因 此混合气体缺氧法和化学缺氧法不能满足高原缺 氧的环境特征。目前, 国内关于高原缺氧细胞模 型的建立尚无统一标准, 尽管利用大型低压氧舱 通过控制压力和氧浓度模拟高原环境建立了动物 高原疾病模型, 但在离体细胞领域仍为空白。本 研究应用低压缺氧装置对细胞进行低压缺氧处理 制备了细胞模型, 旨在为低压缺氧致神经损伤疾 病的机制研究提供可靠的细胞模型。

本文资料显示,在 $1 \%$ 氧浓度、 $41 \mathrm{kPa}$ 的低压 缺氧条件下培养 $24 \mathrm{~h}$ 即可造成细胞损伤。氧是细 胞进行能量代谢的重要原料, 缺氧会影响细胞代 谢进而导致产生大量的氧自由基, 过量氧自由基
会导致氧自由基清除剂 SOD 活性下降。同时,缺 氧过程中细胞会产生大量的活性氧, 与细胞膜中 的不饱和脂肪酸作用,生成大量脂质过氧化产物 MDA, 破坏细胞膜的完整性, 导致细胞膜通透性增 加, 细胞中的 LDH外漏到培养基中 ${ }^{[13-16]}$ 。本文资 料显示, 低压会加重缺氧对细胞造成的损伤, 低压 缺氧组上清液中SOD活性与正常对照组和常压缺 氧组相比明显降低, 说明此时机体内大量的氧自 由基加重了细胞损伤; 同时,低压缺氧组较正常对 照组和常压缺氧组 LDH 活性增强, MDA 含量增 加, 表明低压缺氧进一步造成细胞膜的完整性破 坏。细胞调亡和细胞周期检测结果表明,低压缺 氧可以诱导细胞凋亡, 细胞周期阻滞在 $\mathrm{G} 0 / \mathrm{G} 1$ 期, 但具体机制有待进一步研究。

综上所述, PC12 细胞在 $1 \%$ 氧浓度、 $41 \mathrm{kPa}$ 压 力条件下培养 $24 \mathrm{~h}$ 可以建立低压性缺氧损伤模 型。该模型操作方便、干扰因素较少、可重复性 强, 为深人研究体外神经细胞高原缺氧损伤提供 了便利条件。但高原环境影响因素复杂,除缺氧、 低压外,还包括紫外线、昼夜温差和寒冷等因素, 在后续研究中将继续深人考察相关因素对细胞损 
伤的影响,以便建立更合理的细胞模型。

利益冲突 所有作者均声明不存在利益冲突

\section{参考文献}

[1] ZHANG P, CHEN J S, LI Q Y, et al. Neuroprotectants attenuate hypobaric hypoxia-induced brain injuries in cynomolgus monkeys[J]. Zool Res, 2021, 42(1): 3-19.

[2] SHARMA P, TULSAWANI R. Ganoderma lucidum aqueous extract prevents hypobaric hypoxia induced memory deficit by modulating neurotransmission, neuroplasticity and maintaining redox homeostasis $[\mathrm{J}]$. Sci Rep, 2020, 10(1): 8944.

[3] 陈 建, 黄 缄, 高钰琪, 等. 预缺氧对 PC12 细胞缺 氧后细胞内游离 $\mathrm{Ca}^{2+}$ 的影响 $[\mathrm{J}]$. 西北国防医学杂志, 2008, 29(4): 250-253.

CHEN Jian, HUANG Jian, GAO Yuqi, et al. Effect of hypoxia preconditioning on intracellular $\mathrm{Ca}^{2+}$ of PC12 cells $[\mathrm{J}]$. Medical Journal of National Defending Forces in Northwest China, 2008, 29(4): 250-253. (in Chinese)

[4] 间向丽, 王利胜, 王圣金金. HT22 海马神经元细胞氧糖 剥夺再灌注模型的建立 $[\mathrm{J}]$. 广州中医药大学学报, 2020, 37(181): 161-165.

YAN Xiangli, WANG Lisheng, WANG Shengxin. Establishment of HT22 cell oxygen-glucose deprivation reperfusion model $[\mathrm{J}]$. Journal of Guangdong Pharmaceutical University, 2020, 37(181): 161-165. (in Chinese)

[5] 刘 菲, 张 吴, 刘 博, 等. 氯化钴诱导的 N2a细胞 缺氧损伤模型的机制研究 $[\mathrm{J}]$. 广东药科大学学报, 2020, 36(2): 249-253.

LIU Fei, ZHANG Hao, LIU Bo, et al. Mechanism of cobalt chloride induced hypoxia on N2a cells $[\mathrm{J}]$. Journal of Guangzhou University of Traditional Chinese Medicine, 2020, 36(2): 249-253. (in Chinese)

[6] 李海丽, 卢永昌, 钱 帅, 等. 苍叶雪莲粗提物对 EA. hy926细胞缺氧损伤的保护作用 $[\mathrm{J}]$. 湖北农业科学, 2016, 55(1): 167-169.

LI Haili, LU Yongchang, QIAN Shuai, et al. Saussurea obvallata crude protective effects of hypoxia on EA. hy926 injury [J]. Hubei Agricultural Sciences, 2016, 55(1): 167-169. (in Chinese)

[7] HEIL J, SCHULTZE D, SCHEMMER P, et al. Nacetylcysteine protects hepatocytes from hypoxia-related cell injury [J]. Clin Exp Hepatol, 2018, 4(4): 260-266.

[8] 冯 岗, 张二龙, 高钰琪, 等. 7种不同组织来源细胞缺 氧损伤效应评价 $[\mathrm{J}]$. 第三军医大学学报, 2019, 41(6): 570-574.

FENG Lan, ZHANG Erlong, GAO Yuqi, et al. Evaluation of hypoxic injuries in 7 cell lines derived from different human tissues $[\mathrm{J}]$. Journal of Third Military Medical University, 2019, 41(6): 570-574. (in Chinese)

［9］牛其芳, 李德龙, 杨 杨, 等. 人血管内皮细胞缺氧复 氧损伤细胞模型的建立 $[\mathrm{J}]$. 中国口腔领面外科杂志, 2019, 17(4): 295-299.

NIU Qifang, LI Delong, YANG Yang, et al. Establishment of human vascular endothelial hypoxia/reoxygeneration injury cell model $[\mathrm{J}]$. China Journal of Oral and Maxillofacial Surgery, 2019, 17(4): 295-299. (in Chinese)

［10］刘阳, 安美文, 芦 荣, 等. 压力下角质形成细胞 与成纤维细胞共培养对细胞增殖及胶原合成的影 响 $[\mathrm{J}]$. 医用生物力学, 2015, 30(2): 148-153.

LIU Yang, AN Meiwen, LU Ying, et al. Effects of keratinocytes/fibroblasts co-culture under pressure on cell proliferation and collagen synthesis $[\mathrm{J}]$. Journal of Medical Biomechanics, 2015, 30(2): 148-153. (in Chinese)

［11］黄 正, 刘益杰, 褚立希, 等. 周期性压力通过调亡 基因调节兔软骨细胞增殖和调亡的实验研究 $[\mathrm{J}]$. 国际骨科学杂志, 2014, 35(3): 205-208.

HUANG Zheng, LIU Yijie, CHU Lixi, et al. Periodic pressure regulates the proliferation and apoptosis of rabbit chondrocytes genes $[\mathrm{J}]$. International Journal of Orthopaedics, 2014, 35(3): 205-208. (in Chinese)

[12] COBELENS P M, VAN PUTTE B P, KAVELAARS A, et al. Inflammatory consequences of lung ischemiareperfusion injury and low-pressure ventilation $[\mathrm{J}]$. J Surg Res, 2009, 153(2): 295-301.

[13] TSENG V, NI K, ALLAWZI A, et al. Extracellular superoxide dismutase regulates early vascular hyaluronan remodeling in hypoxic pulmonary hypertension[J]. Sci Rep, 2020, 10(1): 280.

[14] ASANO A, UEDA S, KUJI I, et al. Intracellular hypoxia measured by $18 \mathrm{~F}$-fluoromisonidazole positron emission tomography has prognostic impact in patients with estrogen receptor-positive breast cancer $[\mathrm{J}]$. Breast Cancer Res, 2018, 20(1): 78.

[15] SHI Q, YU C, ZHU D, et al. Effects of dietary Sargassum horneri on resisting hypoxia stress, which changes blood biochemistry, antioxidant status, and hepatic HSP mRNA expressions of juvenile black sea bream Acanthopagrus Schlegelii $[\mathrm{J}]$. J Appl Phycol, 2020, 32(5): 3457-3466.

[16] DEVECI H A, AKYUVA Y, NUR G, et al. Alpha lipoic acid attenuates hypoxia-induced apoptosis, inflammation and mitochondrial oxidative stress via inhibition of TRPA1 channel in human glioblastoma cell line [J]. Biomed PharmacoTher, 2019, 111: 292-304. 